\title{
MODELING THE FORMATION OF AUTOMOBILE BODY PANELS BY AN ELASTIC PUNCH IN THE SOFTWARE PACKAGE PAM-STAMP 2G
}

\author{
A. A. Ershov, ${ }^{1}$ V. V. Kotov, ${ }^{2}$ and Yu. N. Loginov ${ }^{2}$
}

The practice of forming products with the use of an elastic medium has been established for a fairly long time and has shown itself to be a reliable and economic method of making products of complex shape. Stamping with an elastic punch offers the following advantages:

1) the manufacture of products with high mechanical properties and a quality surface;

2) the use of less metal for the production process due to elimination of the need for two tools; and

3) maintenance of high productivity.

Forming with an elastic punch is most often done to make parts for the aerospace sector and to stamp body parts for the automotive industry.

In this article, the software package PAM-STAMP $2 \mathrm{G}$ is used to model the stamping of two large parts of an automobile body - the inside of the hood and the fender (Fig. 1) - by an elastic punch. The results obtained from the modeling are then discussed. The software was developed by the company ESI-Group ${ }^{\circledR}$.

Preparatory operations that make use of the materials and geometry of the products need to be performed before the stamping process can be modeled. The initial geometry is represented by 3D-models of the finished products, and this geometry must then be used to construct the geometry of the tool. These tasks were accomplished by means of the program's internal module Diemaker. The first step entailed closing all the openings, removing the fastenings, and bending the edges of the metal. In the next step, we manually constructed the surface under the clamp, determined the depth of the die, and identified the auxiliary surfaces and fillets between the impression and the surface of the clamp (the tool elements designed for the fender are shown in Fig. 2). The resulting tool (die), in the form of a reticular geometry, was then transferred to the main design module.

The reticular finite-element model of the elastic punch used for both products was constructed in the geometry editor Visual-Mesh (ESI-Group ${ }^{\odot}$ ). A network of three-dimensional elements was used for the given tool. The size of the finite elements of the model was chosen in such a way as to allow all of the fillets, bendings, and radii in the model of the die to be described with a high degree of accuracy during the modeling operation. Here, it was taken into account that the smaller the dimensions of the elements, the longer the computing time. The models were then imported into the design module of PAM-STAMP.

The semifinished products to be used in the stamping operation need to be created in the design module. This was done by using the tool Blank editor - the geometric parameters of the semifinished products were entered into the program through this editor and the material of the semifinished products was chosen. The thickness of the semifinished products was $1.0 \mathrm{~mm}$ for the fender and $0.7 \mathrm{~mm}$ for the inside of the hood. Standard carbon steel was chosen as the material of the semifin-

\footnotetext{
${ }_{1}^{1}$ PLM Ural - Delkam-Ural Companies Group.

${ }^{2}$ Yeltsin Ural Federal University (UrFU).
}

Translated from Metallurg, No. 9, pp. 33-35, September, 2012. 


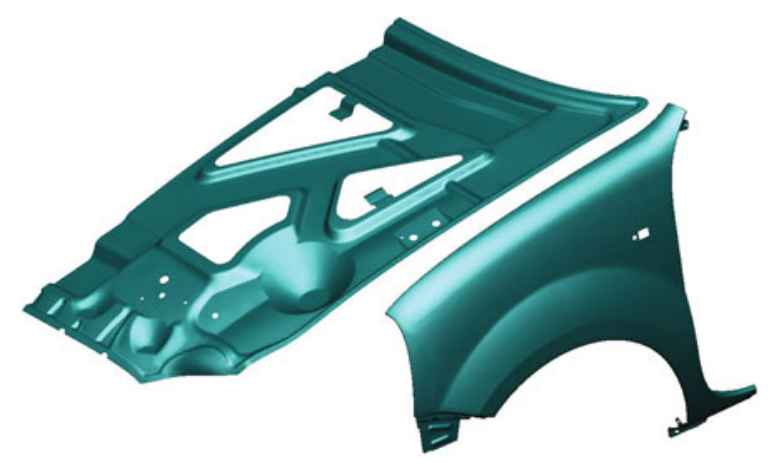

Fig. 1. Exterior view of the finished products: parts of the hood (on the left) and fender (on the right) of an automobile after machining.

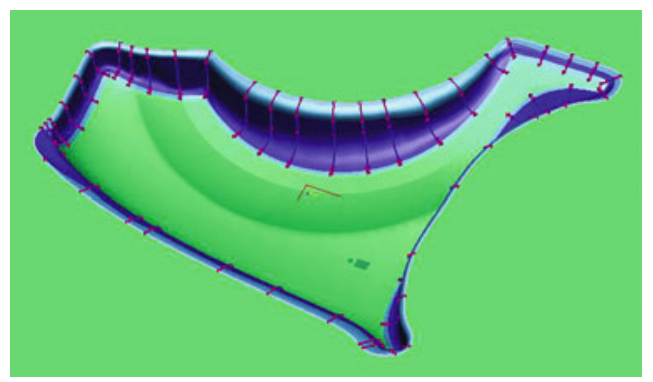

Fig. 2. Design of tool for stamping the fender part in the Diemaker module.

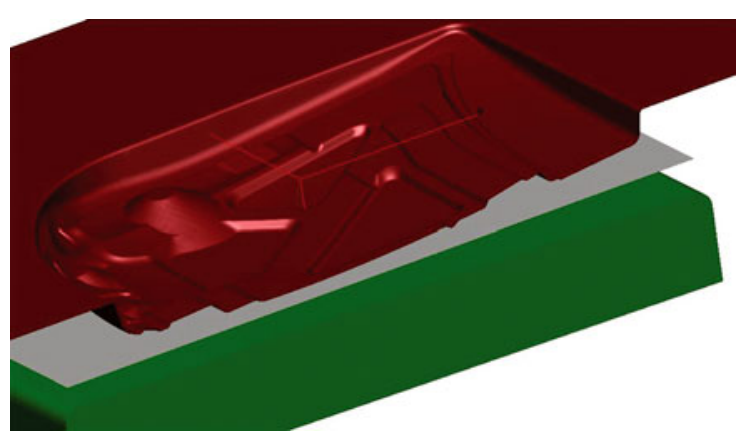

Fig. 3. Exterior view of the assemblage of models of the die, semifinished product, and punch for forming the inside part of the hood.

ished products. Values were specified for the parameters that characterize the orthotropic anisotropy of this steel. The Lankford anisotropy coefficients in three directions were obtained from tensile tests: $r_{0}=1.33 ; r_{45}=1.36 ; r_{90}=1.69$. Figure 3 shows the final form of the assemblage of models of the die, the elastic punch, and the semifinished product for the hood.

After all of the above objects have been created, the problem of shaping the products is formulated: the boundary conditions are determined along with the process parameters. The parameters of the material for the elastic dies of both products were assigned in accordance with the Mooney-Rivlin model built into the program (Fig. 4). The density of the material is $1500 \mathrm{~kg} / \mathrm{m}^{3}$, the Poisson's ratio is 0.499 , and the coefficients for the Mooney-Rivlin law (determined empirically for the given material) are $A=0.009$ and $B=0.005$. 


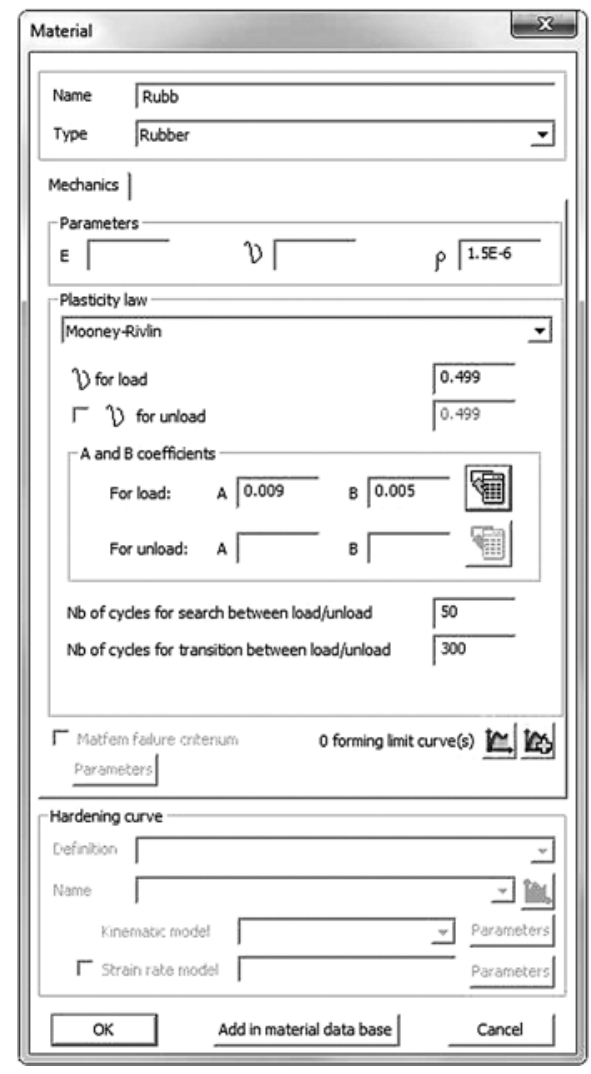

Fig. 4. Specification of the parameters of the elastic material.
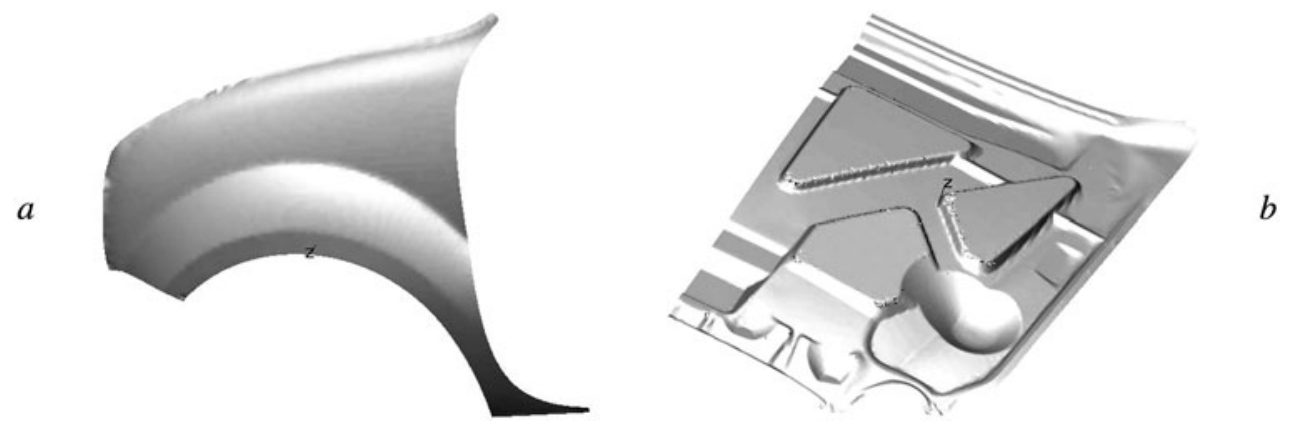

Fig. 5. Exterior view of the fender $(a)$ and hood interior $(b)$ products after modeling of the operations used to form them.

A perfectly rigid body was chosen as the model for the dies. The value chosen for the maximum number of subdivisions of the elements representing the semifinished products was 2 . The friction coefficients for the contact surfaces were determined in accordance with Coulomb's law. In both problems, the friction coefficient is 0.20 on the contact surface between the semifinished product and the elastic punch and 0.12 on the contact surfaces between the die and the semifinished product and between the die and the punch.

The computation is begun after specification of the punch's speed and trajectory and the parameters for stoppage of the operation. The computation is performed by parallel processing with use of the standard solver AUTOSTAMP.

The cutting operation was modeled after the modeling of the shaping operation was completed (Fig. 5). Figure 6 shows the shapes of the elastic punches at the end of the deformation process. 

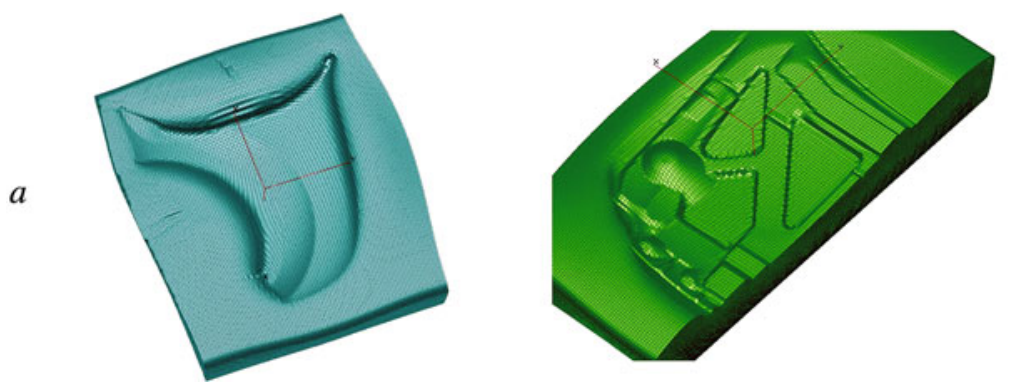

$b$

Fig. 6. Shapes of the elastic fender-forming punch $(a)$ and elastic hood-forming punch $(b)$ at the end of the deformation process.
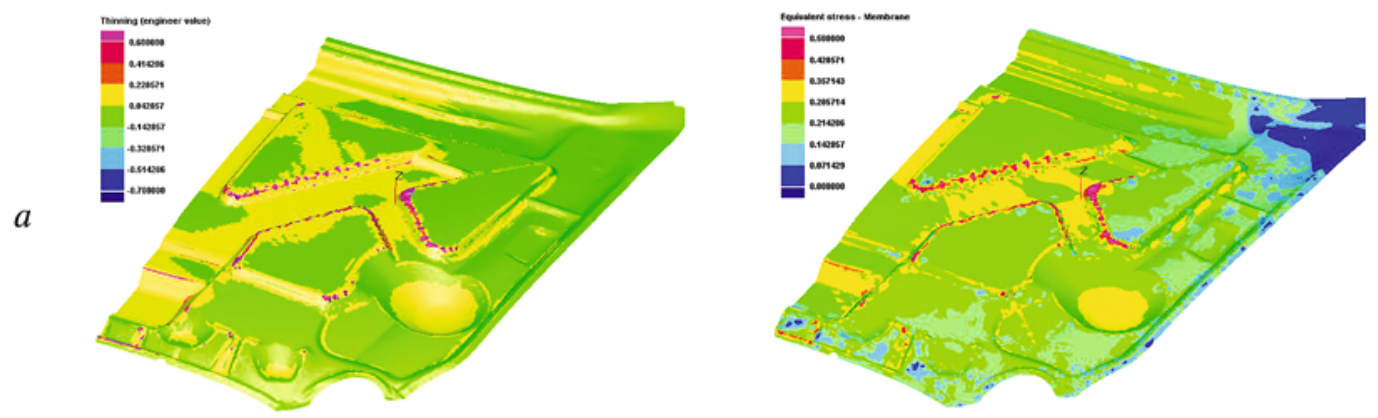

Fig. 7. Fields of the distributions of the thickness reductions in percents $(a)$ and the equivalent stresses in $\mathrm{GPa}(b)$ for the hood product.
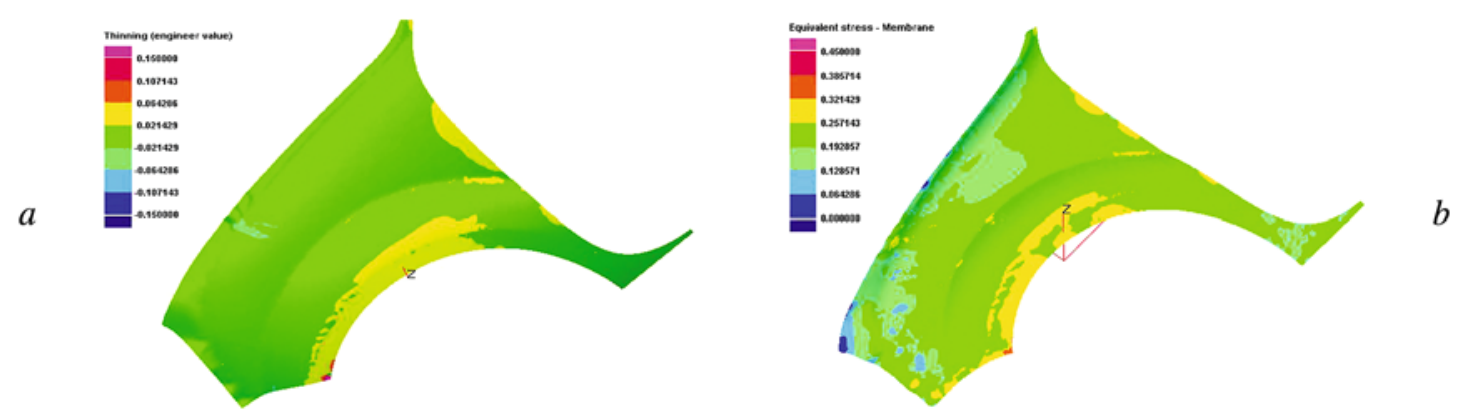

Fig. 8. Fields of the distributions of the thickness reductions in percents $(a)$ and the equivalent stresses in $\mathrm{GPa}(b)$ for the fender product.

$a$

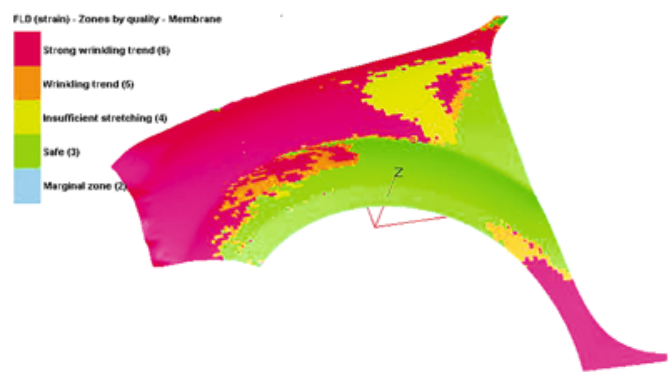

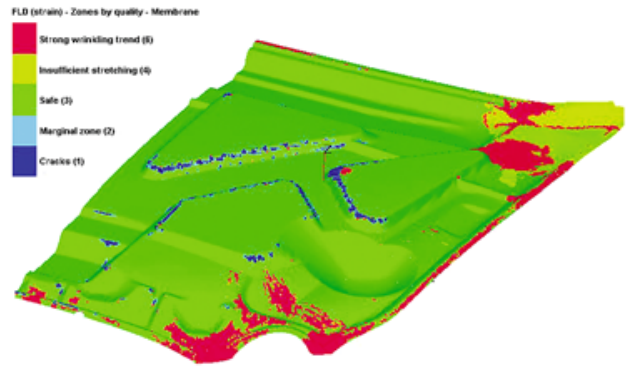

Fig. 9. Qualitative evaluation of the condition of the products' surface based on an analysis of the limiting formability diagrams of the fender $(a)$ and the hood $(b)$. 
It should be pointed out that the parts in question were fully formed. We examined certain computational fields to evaluate the products' formability. Figure $7 a$ illustrates the reduction in the thickness of the inside of the hood. The largest reduction made from the original thickness is $62 \%$ : the thickness of the semifinished product is decreased substantially at the sites where the ribs of the product's interior elements are formed (Fig. 7a). The level of the equivalent stresses in the product is shown in Fig. $7 b$. It can be seen that a high stress level is also reached at the sites just indicated: the maximum value is $543 \mathrm{MPa}$.

The reduction in thickness turned out to be smaller on the fender than on the hood: the maximum reduction was approximately $20 \%$ of the original thickness (Fig. $8 a$ ). For the most part, the metal of this product is of uniform thickness. An evaluation of the level of the equivalent stresses in this product shows that its maximum value is lower than the analogous index for the hood and is roughly $480 \mathrm{MPa}$ (Fig. 8b). Thus, a more favorable stress-strain state is obtained after the stamping of the fender.

The sites where folds and cracks might form in the products can be predicted by analyzing diagrams that describe their limiting formability (by using Keler's model). It was determined that folds (red color) are likely to develop on the surface of the hood's interior (Fig. 9a) in the areas that are shaped last by the die. Also, cracks (blue color) may form in this product in the areas that undergo large reductions in thickness. No potential crack sites were found in the fender, but it was determined that folds are very likely to form on this product. The probability is especially high in the upper part of the fender (Fig. 9b), which needs to be subjected to greater tension.

Thus, the following conclusions can be made. The software package PAM-STAMP 2G has special tools and material models that make it possible to assign the parameters for modeling the operation of shaping with an elastic medium. Modeling the process of stamping the two above parts provided data on the level of the equivalent stresses in the semifinished product, the reductions made in its thickness, and defects that might be formed according to the limiting formability diagrams of the parts. All of this information together makes it possible to determine whether or not the proposed technology is viable. Engineers and technologists can make changes to the technology and the design of the dies and perform real-time evaluations of the expediency of their modifications. 